\title{
Modelling the concentration of antioxidant BHT migrating from polyethylene pipe to water
}

\author{
M. Widomski ${ }^{1}$, B. Kowalska ${ }^{1}$, D. Kowalski ${ }^{1}$, M. Kwietniewski $^{2}$ \\ \& J. Czerwiński ${ }^{1}$ \\ ${ }^{I}$ Faculty of Environmental Engineering, \\ Lublin University of Technology, Poland \\ ${ }^{2}$ Department of Environmental Engineering, \\ Warsaw University of Technology, Poland
}

\begin{abstract}
This paper covers laboratory and modelling studies of antioxidant BHT (butylated hydroxytoluene) migration from HDPE pipe used in water supply systems. Our laboratory experiments were conducted on a measuring stand constructed on a new HDPE pipe. Determination of BHT concentration in pipe samples was performed with high-resolution size-exclusion chromatography (HR-SEC). Concentration of BHT migrating to drinking water in laboratory conditions was determined with a Trace-Ultra/Polaris Q (Thermo USA) GC-MS system. Modelling studies concerning BHT concentration in water body were performed using commercial CDF software Fluent, Ansys Inc. Our calculations were based on experimentally determined input data, such as diffusion coefficient, as well as initial and boundary conditions. The obtained modelling results showed good agreement with the experimental data

Keywords: HDPE pipe, antioxidant BHT migration, numerical modelling, diffusion coefficient, $C D F$.
\end{abstract}

\section{Introduction}

HDPE pipes are commonly used in water supply systems. Nevertheless, substances such as additional components introduced into polymer during its synthesis and processing, their degradation products as well as degradation products of polymer itself, may migrate from pipes into water. These compounds 
may lead to deterioration of water quality, especially its taste and odour. The pipes mechanical properties, their long-term strength in particular, are influenced by the removal of stabilisers. The research on organic compounds migration from plastic pipelines to water flowing through the pipes has been conducted by numerous researchers [1-8]. The most common pollutants originated from polyethylene pipes migrating to drinking water and influencing its quality and organoleptic properties are the following: antioxidants - Irganox 1010, Irganox 1035, Irganox 1076, 2,6-di-tert-butyl-4-methyl phenol (BHT), products degradation of antioxidants used in polymer production - 4-ethylphenol, 4- $t$-butylphenol, 2,6-di-t-butyl-p-benzoquinone, 2,4-di-t-butylphenol, 3,5- di-tbutyl-4-hydroxy styrene, 3,5- di- $t$-butyl-4-aceto phenone, 3,5- di-t-butyl-4hydroxyaceto phenone, 1,5-bis( $t$-butyl)-4-(2-carboxyethylidene) cyclohexa-1,4dien-6-on, 3-(3,5-di-t-butyl-4-hydroksyphenyl) methyl propanoate, 3-(3,5-di- $t$ butylo-4-hydroksyphenyl) propanoic acid, esters - butyl acetate, ethyl hexanoate, hexyl acetate, propyl hexanoate, butyl hexanoate, ethyl octanoate, hexyl hexanoate, hexamethyl butanoate, ethyl decadienoate, 2,2,4-trimethyl-1,32,2,4-TPD, aldehydes - nonanal, decanal, ketones - 2-decanone, 2-undecanone, 2-dodecanone, aromatic hydrocarbons - benzene, toluene, styrene, ethyl benzene, isopropyl benzene, n-propyl benzene, 1,3,5-trimethyl benzene, 1,2,4trimethyl benzene, p-isopropyl toluene, naphthalene.

Migration of stabilisers and other pollutants in PE pipes begins in the polymer matrix and moves towards the surface exposed to water. It depends on the properties and type of migrating component as well as temperature. Migration rate increases with the growth of temperature. In addition, polymer thermodynamic properties, such as polarity or solubility, influence the rate of migration as well.

Water quality modelling has gained on popularity in the last few years, as it allows predicting pollution dispersion in water supply systems in different working conditions of the system. The modelled pollutants may be introduced into the system in the source (water treatment plant e.g. the disinfectant) or in the water supply system e.g. from the pipes constituting the system [9]. Pollution dispersion modelling helps understand the movement and behaviour of pollutants, and thus aids adequate design and management of the network. Majority of migration processes can be described with Fick's diffusion equation. The main aim of migration prediction is to establish mathematical model parameters, which are characteristic of a given combination: migrating component-polymer-medium. The models predominantly require 2 parameters: diffusion coefficient and partition coefficient. Diffusion coefficient represents the rate of migration and partition coefficient represents migration component concentration in relation to the component concentration in the medium in the state of equilibrium. CFD methods (computational fluid dynamics) allow calculating pollution dispersion in water pipes in different flow conditions taking into consideration chemical reactions occurring in water and on pipe walls and their kinetics [5, 10].

One of the most popular commercial CFD software applied in water quality calculations is Fluent, which was required by Ansys in 2006. Fluent is frequently 
reported to be a successful tool in various simulation calculations of fluid flow [11-15]. Fluid flow modelling in CFD is based on the following governing equations e.g. [16, 17]: conservation of mass, conservation of momentum, Navier-Stokes equation. These equations, with the proper set of computational simplifications, boundary conditions and support of numeric techniques, provide comprehensive modelling tool for various laminar and turbulent, compressible and incompressible fluid, steady state and transient fluid (gas, liquid or multiphase) flows analyses. Qualitative calculations in CDF covering transport and mixing of reactive and non-reactive chemical species are usually based on the considered species conservation equations.

The main goal of the research presented in the article was to conduct numerical calculations of changes in the concentration of antioxidant BHT migrating from HDPE pipe, commonly used in water supply systems, into water, using Fluent software. Concentration of BHT in pipe material was determined using high-resolution size-exclusion chromatography (HR-SEC) and innovative research methodology. In order to determine both the BHT concentration in samples of water in contact with pipe material and diffusion coefficient of antioxidant in water, a special measuring stand and a multi-channel gas chromatograph coupled with a mass spectrometer Polaris Q were used.

\section{Experimental methods}

Tests were conducted in Environmental Analysis Laboratory of Lublin University of Technology Faculty of Environmental Engineering. Basic properties of the HDPE material, after manufacturer, were as follows: density: $955 \mathrm{~kg} / \mathrm{m}^{3}, \mathrm{MFI}_{(190.5)}$ : $0.65 \mathrm{~g} / 10 \mathrm{~min}$, volatiles $0.2 \%$, impact strength (Charpy index) $14.5 \mathrm{~mJ} / \mathrm{mm}^{2}$. The measuring stand was constructed on a new HDPE pipe (diameter $25 \times 2.0 \mathrm{~mm}$, length $150 \mathrm{~m}$ ), straight from the manufacturer. The interior surface of the pipe was equal to $9.896 \mathrm{~m}^{2}$, while the total volume of water in the water system was equal to $51.594 \mathrm{dm}^{3}$. The measuring stand diagram is presented in Fig. 1.

Antioxidant BHT tests were aimed at determining its concentration in both the material of the pipe and the water filling the pipe. BHT concentration in the pipe material was measured with an innovative methodology, the premises of which are detailed below.

Water samples were collected in $60 \mathrm{~mL}$ amber glass vials and stored till analysis at the temperature below $4^{\circ} \mathrm{C}$. Water sample or aqueous standard solution aliquots of $40 \mathrm{~mL}$ were transferred into $60 \mathrm{~mL}$ vials with silicon/PTFE septum (Supelco, Bellefonte, USA) and thermostated at $30^{\circ} \mathrm{C}$. Fibre coated with $100 \mu \mathrm{m}$ of PDMS (Supelco, Bellefonte, USA) SPME device was used for the extraction of BHT. The fibre was placed in the head-space of samples for $30 \mathrm{~min}$. Afterwards the fibre was immediately transferred to the PTV injection port of GC-MS system, desorption time $10 \mathrm{~min}$.

Pipe samples (ca $300 \mathrm{mg}$ ) were placed in $5 \mathrm{~mL}$ reaction vials and aliquot of $3 \mathrm{~mL}$ of tetrahydrofurane -THF (HPLC-grade, POCh - Poland) was added. After sealing vials were heated at $60^{\circ} \mathrm{C}$ for $3 \mathrm{~h}$. After cooling extracts were filtrated 


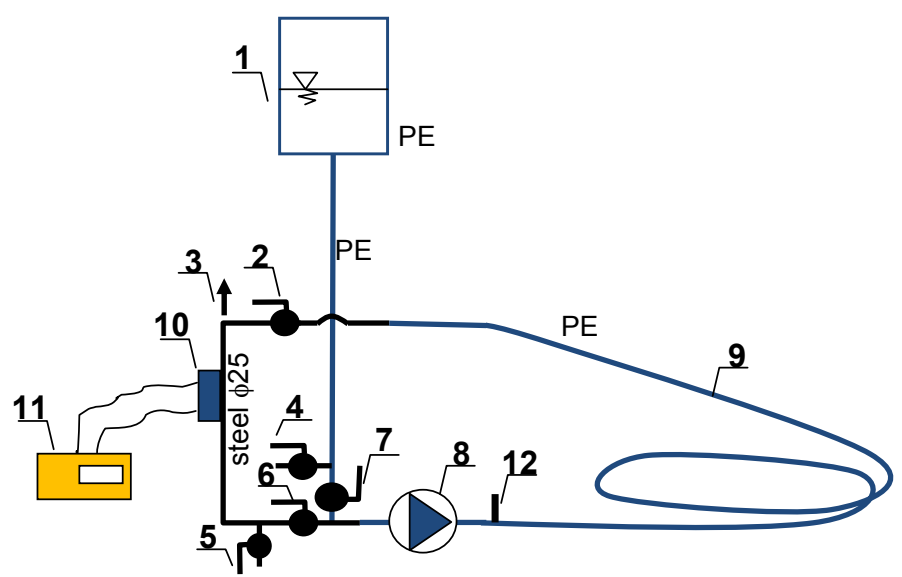

Figure 1: Measuring stand; 1 - PE surge tank, 2 - valve, 3 - deaerator, 4 - drain valve, 5 - tap valve (sampling point), 6 - flow regulating valve $\phi 25 \mathrm{~mm}, 7$ - inlet valve $\phi 25 \mathrm{~mm}, 8$ - Wilo MVIE 203$1 / 16 / \mathrm{E}-3-2 / 8$ centrifugal pump with integrated frequency converter, 9 - tested HDPE pipe, 10 - ultrasonic sensors, 11 - PortaFlow 300 ultrasonic flowmeter, 12 - thermometer.

with Millex PVDF $0.22 \mu \mathrm{m}$ syringe filters. $2 \mathrm{~mL}$ of filtrated extract was injected to the semi-preparative HPLC system (Breeze 1525 - Waters) equipped with Envirogel (Waters - USA) columns, for clean-up.

Extract purification: Purification was performed with high-resolution sizeexclusion chromatography (HR-SEC) with a liquid chromatograph Breeze 1525 (Waters - USA) consisting of the following elements: binary gradient pump Waters 1525, injector - Rheodyne with $2000 \mu$ l sample loop, columns: envirogel GPC cleanup 19x150 mm and envirogel GPC cleanup 19x300 mm, two-wave, tunable detector UV-VIS Waters M2487, fraction collector - Waters FC III, data acquisition and system control: Breeze 3.30SPA software, chromatographic syringes with non-stick pistons $-2500 \mu \mathrm{l}$ (Hamilton).

The system was calibrated for standard solution containing: BHT in THF. Mobile phase (tetrahydrofurane) flow rate $-5 \mathrm{ml} / \mathrm{min}$ and wave length during chromatograms collection $-\lambda=254 \mathrm{~nm}$.

In case of potential influence of column overload protection collection was made for the following elution times - 10-17 minutes.

SEC chromatography is typically used for fractionation of polymers by chain length [18] or for fractionation of environmental or biological extracts for determination of PAH [19], organochlorine pesticides [20], or as a first-step clean-up for the determination of PCDD/Fs [21]. Nevertheless, this methodology has never been used for the analysis of plastic additives.

Appropriate fraction containing BHT was evaporated under gentle nitrogen stream. The residue was dissolved in hexane and injected to the GC-MS system. Injection volume $2 \mu \mathrm{L}$. 


\subsection{GC-MS final analysis}

A Trace-Ultra/Polaris Q (Thermo USA) GC-MS system was applied for final determinations. The injector temperature was held constant at $270^{\circ} \mathrm{C}$. The $\mathrm{GC}$ oven temperature was held for $2 \mathrm{~min}$ at $75^{\circ} \mathrm{C}$, then increased to $270^{\circ} \mathrm{C}$ at $5^{\circ} \mathrm{C} / \mathrm{min}$, and then held at $270^{\circ} \mathrm{C}$ for $10 \mathrm{~min}$. A60 $\mathrm{m} \mathrm{x} 0.25 \mathrm{~mm}$ ID with a $0.25 \mu \mathrm{m}$ thickness of $5 \%$ diphenyl - 95\% dimethyl polysiloxane crossbonded phase (DB-5) capillary column was used, with research grade helium $(99.9996 \%)$ as the carrier gas under a constant flow of $40 \mathrm{~cm} / \mathrm{s}$, in the splitless mode. The MS (ion trap) was operated in the scan mode from $\mathrm{m} / \mathrm{z} 40$ to 350 . BHT was quantified by measuring areas of tree most abundant ions $(\mathrm{m} / \mathrm{z} 205$, $220,177)$.

The limits of quantitation were established at the level of $11 \mathrm{ng} / \mathrm{dm}^{3}(\mathrm{~S} / \mathrm{N}=7)$ for water samples and $23 \mu \mathrm{g} / \mathrm{kg}$ for pipe samples $(\mathrm{S} / \mathrm{N}=7)$.

GC-MS chromatogram extracts of pipe samples are shown in Fig. 2.

Results of our determination show high precision of this method (relative standard deviation in analytical series varied from $1.1 \%$ till $2.1 \%$. Also FSnedecore Test shows that there are no differences between results in a 3 determination series. Recoveries of BHT for presented methodology varied from 95.7 to $102 \%$. This analysis was carried out by standard additions method (because of lack on the market standard reference materials).

Immediately after transporting the HDPE pipe into the laboratory two fragments were removed in order to determine the BHT content in the pipe,



Figure 2: GC-MS chromatogram extracts of pipe samples. Black line - TIC chromatogram extract of whole material; red line - chromatogram on $\mathrm{m} / \mathrm{z} 205$ ion the same sample; blue line - chromatogram on $\mathrm{m} / \mathrm{z}$ 205 ion of extract internal surface layer. 
using the method described above. The samples were collected from the inner surface and the middle of both analysed pipe fragments (whole material). The measurements were repeated three times and the results are presented in Table 1.

Table 1: $\quad$ BHT antioxidant concentration in the HDPE pipe.

\begin{tabular}{|c|c|c|}
\hline BHT mg/kg & Average (3 samples) & Standard dev. \\
\hline P1 inner surface & 719 & 14.80 \\
\hline P1 whole material of the pipe & 640.33 & 15.31 \\
\hline P2 inner surface & 725.00 & 6.56 \\
\hline P2 whole material of the pipe & 601.33 & 9.02 \\
\hline
\end{tabular}

The remaining pipe provided the base for the measuring system, which was then flushed with deionised water, prepared with Milli-Q apparatus (Millipore, Molsheim, Germany), at $2.0 \mathrm{~m} / \mathrm{s}$, so that the water in the system is replaced three times. The aforementioned are standard conditions for activating domestic installations. Basic parameters of the deionised water used for flushing and filling the system were as follows: TOC $\leq 0.5 \mathrm{ppb}$, conductivity $18.2 \mathrm{M} \Omega \mathrm{cm}$. After flushing the system was deaerated and water flow was initiated at the velocity of $1.0 \mathrm{~m} / \mathrm{s}$. As a consequence of employing a closed-loop water flow system, the water temperature increased and stabilised after 14 hours at $33^{\circ} \mathrm{C}$. At that time a water sample for BHT concentration measurement was collected. Subsequent samples were collected after 24, 36, 48 and 72 hours, which aims to reflect a typical water age in water supply networks [22]. Each sample collection procedure required exchanging $0.230 \mathrm{dm}^{3}$ of water in the system. BHT concentration values are presented in Table 2.

Table 2: $\quad$ BHT concentration in the water from the measuring stand $\mathrm{g} / \mathrm{m}^{3}$.

\begin{tabular}{|c|c|c|c|c|c|c|}
\hline \multirow{2}{*}{ Hours } & \multicolumn{3}{|c|}{ Sample No., } & \multirow{2}{*}{ Average } & $\begin{array}{c}\text { Standard } \\
\text { dev. }\end{array}$ & $\begin{array}{c}\text { Temp. } \\
{ }^{\circ} \mathrm{C}\end{array}$ \\
\cline { 2 - 4 } & $\mathrm{Nr} 1$ & $\mathrm{Nr} 2$ & $\mathrm{Nr} 3$ & & & 18,2 \\
\hline 0 & $\mathrm{ND}$ & $\mathrm{ND}$ & $\mathrm{ND}$ & - & - & - \\
\hline 6 & $\mathrm{ND}$ & $\mathrm{ND}$ & $\mathrm{ND}$ & - & - & 23,2 \\
\hline 14 & $1,4 \mathrm{E}-05$ & $1,2 \mathrm{E}-05$ & $1,3 \mathrm{E}-05$ & $1,300 \mathrm{E}-05$ & $1,00 \mathrm{E}-06$ & 33,0 \\
\hline 24 & $6,9 \mathrm{E}-05$ & $7,6 \mathrm{E}-05$ & $7,2 \mathrm{E}-05$ & $7,233 \mathrm{E}-05$ & $3,51 \mathrm{E}-06$ & 33,1 \\
\hline 36 & $1,38 \mathrm{E}-04$ & $1,49 \mathrm{E}-04$ & $1,43 \mathrm{E}-04$ & $1,433 \mathrm{E}-04$ & $5,51 \mathrm{E}-06$ & 33,1 \\
\hline 48 & $2,09 \mathrm{E}-04$ & $2,11 \mathrm{E}-04$ & $2,23 \mathrm{E}-04$ & $2,143 \mathrm{E}-04$ & $7,57 \mathrm{E}-06$ & 32,9 \\
\hline 72 & $2,13 \mathrm{E}-04$ & $1,92 \mathrm{E}-04$ & $2,17 \mathrm{E}-04$ & $2,073 \mathrm{E}-04$ & $13,43 \mathrm{E}-06$ & 33,1 \\
\hline
\end{tabular}

$\mathrm{ND}$ - not detected

\subsection{Determination of diffusion coefficient value for BHT}

Determining the value of diffusion coefficient for BHT migrating from HDPE pipe into water was performed on BHT experimentally determined concentration values and Fick's equation, according to the algorithm presented in Fig. 3. The calculations were carried out in Excel. 


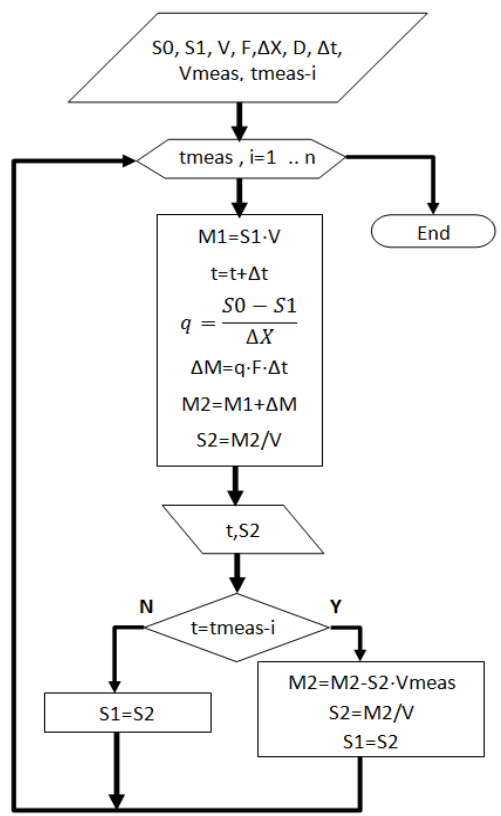

where:

$\mathrm{S} 0$ - BHT concentration in pipe material, $\mathrm{S} 1, \mathrm{~S} 2$ - BHT concentration in water, $\mathrm{V}$ - water volume in the system, $\mathrm{F}$ - inner surface of the pipeline, D - diffusion coefficient, $\Delta \mathrm{t}$ - time step, $\Delta \mathrm{X}$ - diffusive layer thickness, Vmeas - collected sample volume, tmeas - sample collection time, M1, M2 -BHT in water mass, $\mathrm{q}-\mathrm{BHT}$ diffusion flux,

Figure 3: Block diagram of BHT concentration determination algorithm in constant-flow closed-loop measuring system.

Several simplifying assumptions were made and used the calculations: constant BHT concentration in pipe material, constant temperature of $33^{\circ} \mathrm{C}$, measurements commenced after 14 hours into the experiment, which was the time required for the temperature to stabilise at $33^{\circ} \mathrm{C}$, constant flow velocity of $1 \mathrm{~m} / \mathrm{s}$, time step equalled time required for one circulation cycle of water in the system $(150 \mathrm{~s})$, diffusive layer thickness derived from literature and equal to $100 \mu \mathrm{m}[23,24]$. Calculations were conducted with the method of successive approximations, adjusting the diffusion coefficient values. The results of calculations are presented in Table 3.

Conducted simulations of diffusion coefficient included a series of simplifying assumptions, to mention assumed diffusive layer thickness, based on literature data, which might have had influence on the quality of obtained results. Another element of potential influence on the interpretation of calculations results was a relatively high temperature of water in the measuring system when measurements were conducted. Lowering the temperature to most common conditions in existing water supply networks and water supply systems would require modifications of the measuring stand and introduction of a cooling system. Despite the aforementioned simplifying assumptions, the simulation calculations presented in Table 4 indicate their high compliance with the values derived from measurements. The percent matching error did not exceed $0.2 \%$. A noticeable difference appeared in the case of the last result (72nd hour of measurement, 58th hour of simulation). The calculated percent matching error amounted to over $71 \%$. We believe that such discrepancy between the 
measurement and calculation results is a consequence of simulation numerical model failing to include BHT decay in water. Literature indicates that BHT halflife time for the first order hydrolysis, determined in test conditions by Gandek et al. [25], equals $22 \pm 6$ days at $20^{\circ} \mathrm{C}$. Obtained results appear to indicate that whenever the water age of 48 hours is exceeded, this factor should be taken into consideration. The calculated diffusion coefficient value provided base for numerical computations of BHT concentration changes when exposed to HDPE pipe.

Table 3: $\quad$ Results of calculations.

\begin{tabular}{|c|c|c|c|c|}
\hline \multicolumn{2}{|c|}{ Hour of } & \multicolumn{2}{|c|}{ BHT concentration, $\mathrm{g} / \mathrm{m}^{3}$} & \multirow{2}{*}{$\begin{array}{c}\text { Percent matching } \\
\text { error, } \delta \text { (relative } \\
\text { error) }\end{array}$} \\
\hline $\begin{array}{c}\text { measureme } \\
\mathrm{nt}\end{array}$ & simulation & measurement & calculation & \\
\hline 0 & & $0,000 \mathrm{E}+00$ & & \\
\hline 14 & 0 & $1,300 \mathrm{E}-05$ & \multicolumn{2}{|c|}{ Calculations initiation } \\
\hline 24 & 10 & 7,233 E-05 & 7,232 E-05 & $0,019 \%$ \\
\hline 36 & 22 & 1,433 E-04 & 1,433 E-04 & $0,104 \%$ \\
\hline 48 & 34 & 2,143 E-04 & 2,147 E-04 & $0,171 \%$ \\
\hline 72 & 58 & 2,073 E-04 & 3,564 E-04 & $71,907 \%$ \\
\hline \multicolumn{4}{|c|}{ Calculated diffusion coefficient, $\mathrm{m}^{2} / \mathrm{s}$} & $1,337 \mathrm{E}-15$ \\
\hline
\end{tabular}

\subsection{Numerical model}

The presented preliminary three-dimensional and transient numerical modelling of BHT migration and distribution inside the water body flowing with the constant mean flow velocity was conducted with commercial computational software Fluent 6.3, Ansys 12.1 Ansys Inc. Fluent is standard software, established and frequently applied in science and engineering, based on the finite element/volume method and belongs to a still developing group of Computational Fluid Dynamics (CFD) models. The modelled domain in our calculations reflects the water body of a closed loop constructed of $25.0 \times 2.0 \mathrm{~mm}$ HDPE pipe of $150 \mathrm{~m}$ length. The developed three-dimensional model consisted of 635112 nodes and 537959 elements.

Our modelling attempt contains several simplifications. The developed finite element mesh model does not reflect the circulation pump - the modelled movement of the whole water body with constant flow velocity was achieved by assigning the constant value of axial velocity to the selected small volume of fluid, transferring the movement to the rest of the model domain. The changes in BHT concentration caused by water sampling to chemical analyses and adding pure distilled water to fill the volume of laboratory installation were not reflected in our calculations due to their negligibly low value. Our model does not reflect the decay reaction of BHT in water either. 
Time duration of our simulation covered 58 hours of constant flow inside the closed-loop pipe with mean flow velocity of $1.0 \mathrm{~m} / \mathrm{s}$. The standard, twoequations, k- $\varepsilon$ turbulence model with standard wall functions was applied to our modelling calculations of viscous, incompressible liquid flow. The value of pipe wall roughness equal to $1 \cdot 10^{-5} \mathrm{~m}$ was applied. Simulations were performed for water of temperature 32.6 degrees $\mathrm{C}$, density $994.25 \mathrm{~kg} / \mathrm{m}^{3}$, dynamic viscosity $7.2974 \cdot 10^{-4} \mathrm{~kg} /(\mathrm{m} \cdot \mathrm{s})$ and specific heat equal to $4069.1 \mathrm{~J} /(\mathrm{kg} \cdot \mathrm{K})$. The molecular mass of BHT applied in modelling was equal to $220.35 \mathrm{~g} / \mathrm{mol}$, while assumed value of diffusion coefficient for BHT in water, according to our own measurements and calculations, was equal to $1.337 \cdot 10^{-15} \mathrm{~m}^{2} / \mathrm{s}$. The initial condition of BHT concentration was described as pollutant mass fraction in $\mathrm{t}=0$ equal to $1.307 \cdot 10^{-11}$ (concentration $1.30 \cdot 10^{-5} \mathrm{~g} / \mathrm{m}^{3}$ ). The required boundary condition for BHT mass transport on the limit of water body (border between water body and pipe material) was assumed as first type, time-constant Dirichlet condition described as a specific BHT mass fraction equal to $1.2581 \cdot 10^{-15}$ (concentration $1.2514 \cdot 10^{-09} \mathrm{~g} / \mathrm{m}^{3}$ ). The value of BHT mass fraction applied as the boundary condition, reflecting the concentration of BHT migrating from plastic pipe to a thin layer covering pipe wall was determined by a trial and error method.

Calculations were performed for the constant length of time step equal to $60 \mathrm{~s}$. Results of our calculations covered spatial, 3D distributions of, inter alia, flow velocity magnitude, axial and radial velocity, turbulence energy and intensity as well as BHT mass fraction.

\section{Results}

Analyzed results of our preliminary simulation of antioxidant BHT migration and propagation inside the water body of closed loop covered mainly tested species distribution and time-dependent mean mass fraction and concentration.

Figure 4 presents the flow velocity distribution in the selected part of the modeled laboratory installation as well as result of mean velocity calculations. The good agreement between real and calculated value of mean flow velocity is visible $-1.0 \mathrm{~m} / \mathrm{s}$ vs. $1.000119 \mathrm{~m} / \mathrm{s}$.

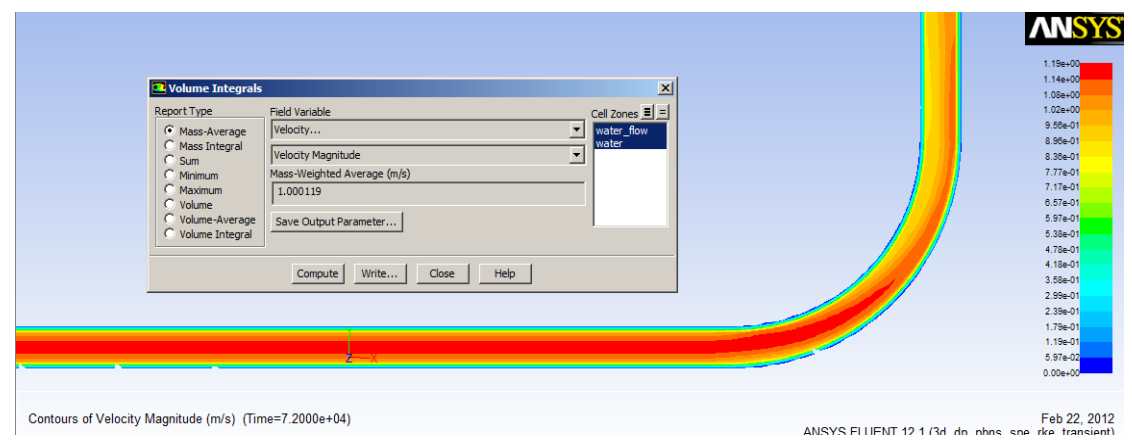

Figure 4: Calculated velocity of flow inside modeled installation. 
Time variability of calculated mean concentration of antioxidant BHT, in comparison to measured values is presented at Fig. 5. The maximum calculated value of mean BHT concentration was equal to $2.0445 \cdot 10^{-04} \mathrm{~g} / \mathrm{m}^{3}$ (mass fraction equal to $2.0554 \cdot 10^{-10}$ ) while the measured value of studied species concentration reached the value of $2.0733 \cdot 10^{-04} \mathrm{~g} / \mathrm{m}^{3}$ (mass fraction $-2.08 \cdot 10^{-10}$ ). So, the values of final concentration are in good agreement, the relative error is equal to $1,39 \%$. But the clear difference in measured and calculated BHT concentration increase during the time of our experiment is also visible. The higher increase of calculated then measured BHT concentration was noted during the period of the first 24 hours of experiment. These differences in calculated and measured values showed that some adjustments to the applied procedure of BHT diffusion coefficient may be necessary. But, despite the mentioned dissimilarity, and taking into consideration that presented calculations should be treated as preliminary, the general fit may be assessed positively - the obtained coefficient of correlation for two compared series is equal to $\mathrm{R}=0.827048$. Moreover, results of our calculations show no BHT decay, while decomposition model was not introduced into the presented simulation.

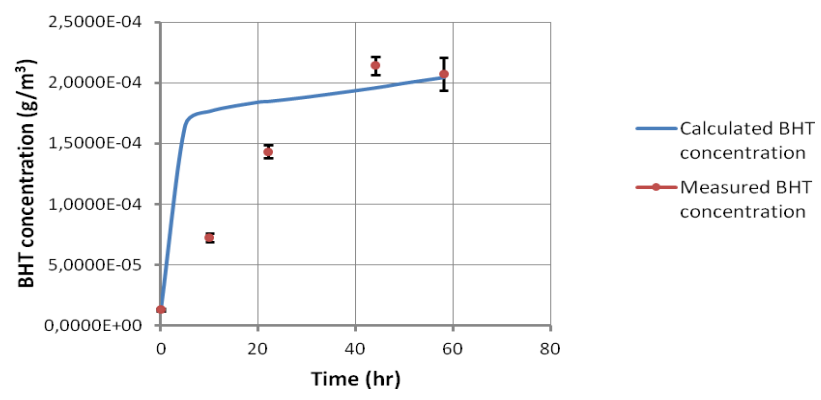

Figure 5: Calculated and measured time-dependant BHT concentration inside the laboratory installation.

\section{Conclusions}

Determining the value of diffusion coefficient for BHT migrating from HDPE pipe into water was performed using simplifying assumptions; nevertheless the simulation calculations indicate their high compliance with the values derived from measurements.

Modelling studies concerning BHT concentration in water body, performed using commercial CDF software Fluent, Ansys Inc. showed good agreement with the experimental data, however the differences in measured and calculated values, especially in the early time steps of simulation, resulting from the assumed modelling simplifications, were observed.

The quality of our results may be improved by introduction of BHT decay reaction and readjusting assumed simplifications during input data calculations. 


\section{References}

[1] Anselme C., N'guyen K., Bruchet A., Mallevialle J., Characterisation of low molecular weight products desorbed from polyethylene tubing. Science of Total Environment, 47, pp. 371- 384, 1985.

[2] Brocca D., Arvin E., Mosbak H.: Identification of organic compounds migrating prom polyethylene pipelines into drinking water. Water Research, 36, pp. 3675-3680, 2002.

[3] Skjevark I., Due A., Gjerstad K.O., Herikstad H.: Volatile organic components migrating from plastic pipes (HDPE, PEX and PVC) into drinking water. Water Research, 37, pp. 1912-1920, 2003.

[4] Schweitzer L., Tomboulian P., Atasi K., Chen T., Khiari D., Utility quick test for analyzing materials for drinking water distribution systems for effect on taste-and-odor. Water Sci. Technol., 49, pp. 75-80. 2004.

[5] Denberg M., Arvin E., Hassager O., Modeling of the release of organic compounds from polyethylene pipes to water. Journal of Water Supply: Research and Technology - AQUA, 56, pp. 435-443, 2007.

[6] Lethola M., J., Miettinen I. T., Keinänen M.M, Kekki T.K, Laine O, Hirvonen A., Vartiainen T., Martikainen P.J., Microbiology, chemistry and biofilm development in a pilot drinking water distribution system with copper and plastic pipes. Water Research, 38, pp. 3769-3779, 2004.

[7] Tomboulian P., Schweitzer L., Mullin K., Wilson J., Khiari D., Materials used in drinking water distribution systems: contribution to taste-and-odor. Water Sci. Technol., 49, pp. 219-226, 2004.

[8] Heim T. H., Dietrich A. M., Sensory aspects and water quality impacts of chlorinated and chloraminated drinking water in contact with HDPE and cPVC pipe. Water Research, 41 pp. 757-764, 2007.

[9] Ratnayake N. and Jayatilake I.N., Study of transport of contaminants in a pipe network using the model Epanet. Water Sci. Technol., 40, pp. 115-120, 1999.

[10] Farmer R., Pike R. and Cheng G., CFD analyses of complex flows. Computers and Chemical Engineering, 29, pp. 2386-2403, 2005.

[11] Norton T. and Sun D.W., Computational fluid dynamics (CFD) an effective and efficient design and analysis tool for the food industry: A review. Trends in Food Science \& Technology, 17, pp. 600-620, 2006.

[12] Ma L., Ashworth P.J., Best J.L., Elliott L., Ingham D.B., Whitcombe L.J., Computational fluid dynamics and the physical modelling of an upland urban river. Geomorphology, 44, pp. 375-391, 2002.

[13] Liu S.X., Peng M., Verification of mass transfer simulation with CFD using highly accurate solutions. Computers and Electronics in Agriculture, 49, pp. 309-314, 2005.

[14] Mohammadi H. and Bahramian F., Boundary Conditions in Simulation of Stenosed Coronary Arteries. Cardiovasc Eng, 9, pp. 83-91, 2009.

[15] Ahmad A.L., Lau K.K., Bakar A., Shukor A., Integrated CFD simulation of concentration polarization in narrow membrane channel. Computers and Chemical Engineering, 29, pp. 2087-2095, 2005. 
[16] Ansys Fluent UDF Manual. Ansys Inc., 2009.

[17] Wesseling P.: Principles of Computational Fluid Mechanics. Springer Series in Computational Mathematics, 29, pp. 167-188, 2000.

[18] Gaborieau M., Castignolles P., Size-exclusion chromatography (SEC) of branched polymers and polysaccharides, Anal. Bioanal. Chem., 399, pp. 1413-1423, 2011.

[19] Fisher S.J., Alexander R., Kagi R.I., Size-exclusion chromatography on zeolites in the trace analysis of polyaromatic hydrocarbons and organochlorine pesticides, J. of Chromatogr. A, 642, pp. 205-209, 1993.

[20] Kim M.-S., Kang T. W., Pyo H., Yoon J., Choi K., Hong J., Determination of organochlorine pesticides in sediment using graphitized carbon black solid-phase extraction and gas chromatography/mass spectrometry. $J$. Chromatogr. A, 1208, pp. 25-33, 2008.

[21] Focant J.-F., Eppe G., Pirard C., Edwin E., Fast clean-up for polychlorinated dibenzo-p-dioxins, dibenzofurans and coplanar polychlorinated biphenyls analysis of high-fat-content biological samples, J. Chromatogr. A, 925, pp. 207-221, 2001.

[22] Kowalski D., Water age in real water pipe-networks. Polish Journal of Environmental Studies, 18, pp. 183-187, 2009.

[23] Siepman J., Peppas N.A, Modeling of drug release from delivery systems based on hydroxypropyl methylcellulose (HPMC). Advanced Drug Delivery Reviews, 48, pp. 139-157, 2001.

[24] Zuleger S., Fassihi R., Lippold B.C., Polymer particle erosion controlling drug release. II. Swelling investigations to clarify the release mechanism. International Journal of Pharmaceutics, 247, pp. 23-37, 2002.

[25] Gandek T. P., Hatton T. A., Reid R. C. Batch extraction with reaction Phenolic antioxidant migration from polyolefins to water.1. Theory.; 2. Experimental results and discussion, Ind. Eng. Chem. Res., 28, pp. 10301036, pp. 1036-1045, 1989. 